\title{
Effect of a Virtual Pharmacy Review Program: A Population Health Case Study
}

\author{
Bushra Muraywid, PharmD, BCPS; Laura E. Butkievich, PharmD, MHA, BCPS; \\ and Brad Myers, PharmD, MBA, BCPS
}

\begin{abstract}
INTRODUCTION: The growing emphasis on value-based health care has created a need for innovative population health management strategies. Pharmacists are underused resources for implementation of meaningful population health models that ensure appropriate medication use through optimization of electronic medical record (EMR) technology and pharmacist knowledge. The objective of our program was to improve the health outcomes of a patient population with diabetes while also reducing costs.

PROGRAM DESCRIPTION: A virtual pharmacy review (ViPRx) program was used to remotely provide previsit comprehensive medication reviews for patients in the defined population. The pharmacist used the EMR to review medications and relevant histories and to intervene when needed to ensure appropriate medication use. Pharmacist recommendations and supporting statements were delivered to the provider's EMR in-box 1-2 days before a scheduled visit. The information technology resources and virtual model allow the pharmacist to manage patient care and collaborate with providers electronically across multiple clinic locations.
\end{abstract}

OBSERVATIONS: The pharmacist managed a panel of over 700 patients in this virtual model. The program has yielded improvements in key diabetes metrics. Most notable is a $6 \%$ increase in the percentage of patients with a hemoglobin $\mathrm{A} 1 \mathrm{c}(\mathrm{HbA1c})$ value of $9 \%$ or less and a $7 \%$ improvement in the controlled low-density lipoprotein (LDL) measure. Monitoring parameters (nephropathy screening, HbA1c, and LDL) increased by $8 \%-12 \%$ from baseline. Additional positive outcomes include improved medication adherence in the defined population as seen by a $1.5 \%$ improvement in medication possession ratio for diabetes medications. Reductions in per member per month (PMPM) prescription costs are estimated at \$11 per month through discontinuation of unnecessary and duplicate medications.

IMPLICATIONS: The results of this case study on the effect of a virtual pharmacy review program demonstrate an opportunity for pharmacists to engage in a population health management model that improves patient outcomes and may reduce the rate at which PMPM prescription drug costs increase.

J Manag Care Spec Pharm. 2020;26(1):24-29

Copyright $\odot 2020$, Academy of Managed Care Pharmacy. All rights reserved.

\section{What is already known about this subject}

Health systems need to develop and implement population health management models in response to value-based payments. As medication experts, pharmacists have the knowledge and skills needed to improve outcomes related to medication use

\section{What this study adds}

A virtual pharmacy review program demonstrated 1 pharmacist's ability to manage a panel of over 700 patients receiving care at 9 different clinics.

Clinical metrics, including the percentage of patients achieving a hemoglobin Alc value $\leq 9 \%$, percentage of patients with a controlled low-density lipoprotein, and medication adherence improved following pharmacist intervention.

Use of a virtual pharmacy review model that leverages information technology resources allows for a broader reach of patients and optimization of pharmacy resources in the ambulatory care setting.

D opulation health has been defined as "the health outcomes of a group of individuals, including the distribution of such outcomes within a group." "The growing emphasis on value-based health care has created a need for innovative population health management strategies. Pharmacists have the knowledge and skills needed to improve outcomes related to medication use. Examples of pharmacist-driven services that have a positive effect on the health of a population include drug information, patient and provider education, disease management, and formulary management. Pharmacists can positively affect patient outcomes through direct patient care. A 2009 meta-analysis of 298 studies concluded that "pharmacistprovided direct patient care has favorable effects across various patient outcomes, health care settings, and disease states." Pharmacist intervention groups lowered hemoglobin Alc (HbAlc) by $1.8 \%$ on average, relative to a comparison group that did not receive direct patient care from a pharmacist. ${ }^{2}$ The pharmacist intervention most frequently cited among studies included in this meta-analysis was patient education, which included medication and disease state education. Additional pharmacist interventions that were reported included medication adherence education, drug utilization review, and chronic disease state management. ${ }^{2}$

One study evaluating the effect of pharmacists providing comprehensive pharmacotherapy workups and medication therapy management (MTM) services found patients were better managed-45\% of patients with diabetes were optimally managed (composite of $\mathrm{HbAlc}$, low-density lipoprotein [LDL], 
blood pressure, aspirin use, and no smoking) compared with $21 \%$ of patients not receiving MTM services. ${ }^{3}$ The same program also demonstrated a reduction in total health expenses for patients receiving MTM of over $\$ 3,500$ annually compared with patients who did not receive MTM services. ${ }^{3}$

Interventions that improve medication adherence among patients may also have significant financial advantages to the health system. A systematic review of 79 studies that assessed the economic burden of medication nonadherence found that medication nonadherence may cost up to $\$ 52,341$ annually per person (with the range being $\$ 5,271-\$ 52,341$ ). ${ }^{4}$

Considering the known effect that pharmacists can have and the need for innovative population health management programs, the pharmacy department of our academic medical center was enlisted to aid physicians in improving the care of a select ambulatory patient population. The objectives were to positively affect clinical and cost-savings measures. The program also tested the effect of an ambulatory care pharmacist who provided clinical recommendations outside the traditional direct patient care model.

\section{Program Description}

The target population consisted of adult patients with diabetes insured by the academic institution's narrow or custom network. The narrow/custom network plan is 1 of 3 plans offered to university employees and dependents, with lower premiums, no deductible, and low copays while limiting choice of providers to those within the academic health system. This group of narrow network patients with diabetes was identified for population health intervention based on rising medication costs, increasingly complicated antidiabetic medication regimens, and risk for diabetic complications with poor disease management.

Because of a large number of patients, initially estimated at 755 , in the population and with 8 primary care clinics spanning 50 miles, a uniquely innovative model was required to address the management of all patients. The traditional model of ambulatory care pharmacy services, where a pharmacist is based in 1 clinic and focuses on chronic disease state management for individual patients through a referral process, would not address this program's need for comprehensive population health management. In addition, it was understood that not every patient would require face-to-face intervention, but it was determined that every patient would benefit from a previsit comprehensive medication review (pvCMR) by a pharmacist to ensure appropriate medication use.

Based on the needs of the target population and desired outcomes, a previsit virtual pharmacy review program called ViPRx (virtual pharmacy review) was created. This program aims to achieve the desired outcomes of improving clinical and cost outcomes through an innovative and efficient model that leverages electronic medical record (EMR) and registry technology to optimize pharmacist resources. Direct patient care services (face to face or by telephone) are not used in this model in order to enhance pharmacist time spent in evaluating medication use and ensuring medication optimization.

Patients in the defined population with upcoming primary care visits at any of the health system's clinics are identified through a daily report electronically sent to the pharmacist via email. The daily report is sorted by visit date and includes the following information: visit number, patient name, appointment date, time and location, provider name, nurse care coordinator name (if applicable), and reason for the visit. The pharmacist uses clinical judgment, visit reason, and a brief evaluation of chart notes and messages to determine if a pvCMR is indicated (e.g., a pvCMR would not be needed if a patient is scheduled for a mole removal). When a pvCMR is indicated, the pharmacist uses a note template within the EMR to complete the review. The note template includes prepopulated patient demographic information and allergy list, medication list, and a list of past immunizations. The pharmacist completes a medication use section, a disease state management section, and a recommendation section.

The pharmacist completes a thorough and extensive chart review of the patient's medication, medical, surgical, and immunization history. All past and current medications are reviewed in detail to ascertain efficacy and tolerability. The pharmacist evaluates all medications, not only those related to diabetes management or those listed on the medication list. The pharmacist uses refill history records, e-messages, and phone messages between the patient and their care team to identify any prescription, over-the-counter, or herbal medications that the patient may be taking but are not included on the medication list. The pharmacist review uses clinical knowledge, any current and relevant guidelines (e.g., American Diabetes Association guidelines), manufacturer prescribing information, and drug references such as Lexicomp to ensure appropriate indication, dosing, and monitoring of all medications. The pharmacist identifies potential duplications of therapy, as well as drug-drug, drug-allergy, and drug-disease interactions if applicable. The comprehensive review assesses for medication nonadherence through payer claim refill history.

The majority of patients use an in-house pharmacy, in which case, the local pharmacy records are assessed to verify refill history. Refill history, as well as chart notes and messages, are used to assist the pharmacist in completing a medication history. Pertinent medication-related concerns that arise from the extensive assessment outlined would be included in the medication use section of the note and ultimately the recommendations section if provider action is needed.

A disease state management assessment is completed with specific attention to evidence-based goals and strategies to achieve those goals. Cost-effectiveness and patient-centered care are promoted by assessing for lower cost alternatives and 
minimizing polypharmacy. To further promote patient-centered care and shared decision making, the pharmacist review and note include an outline of possible treatment options with a discussion of pros and cons, including cost, and when additional therapy may be needed. The pharmacist has access to look up the formulary status and copay for any medication and frequently includes the expected copay for medications that may be needed. A recommendation section follows the disease state management section and summarizes any findings that require provider action.

Reviews are completed and documented 1-2 business days before a scheduled primary care visit and are sent to providers in the EMR. Direct patient care services and telehealth services by the pharmacist are not used. The pharmacist uses the EMR to virtually complete CMRs and sends these reviews to providers electronically at a time when they are best equipped to act on the pharmacist's recommendations-just before a scheduled visit with the patient. For each CMR that is completed, an electronic intervention tracker is logged in the patient's medical record to measure types of interventions, time spent, and physician response to the pharmacist's recommendations. In addition to previsit reviews, the pharmacist is also alerted to electronically prescribed prescriptions for patients in the defined population. The pharmacist reviews prescriptions in real time to ensure appropriate use of medications between comprehensive reviews and in times of care transitions. This unique and innovative model allows the pharmacist to review a greater volume of patients and make more interventions and prevents the patient from needing to make extra visits to the clinic.

\section{Observations}

Implementation of the program required collaboration among an interdisciplinary team of pharmacists, physicians, finance personnel, and information technologists (IT). Patients were identified using the EMR's health registries database and payer data. To determine which patients had diabetes, the diabetes health registry list was used. This list had been previously developed by the population health IT team to assess and compare certain diabetes measures among clinics and providers. The list relied on various criteria such as problem lists and diagnosis codes to determine if a patient had diabetes. The IT team not only played a prominent role early on in identifying eligible patients, but also in formulating worklists, electronic task alerts, reports, note templates, and an electronic intervention tracker.

The program was rolled out to the 8 primary care clinics in a stepwise fashion over 3 months. This staggered roll-out method was used to ensure that any early concerns that arose could be quickly addressed with less effect on all providers and clinics. For example, a need to refine the prescription alert tool to more accurately capture prescriptions written for patients in the population was identified. Also, early program improvements included an effort to improve the accuracy of the diabetes health registry; this was done by identifying and removing patients from the registry who were misidentified as having diabetes.

Based on provider feedback and in an effort to improve the integration of the pharmacist review into the provider workflow, the ViPRx pharmacist formed a relationship with the ambulatory care coordinators (ACCs), a group of registered nurses based in the clinics assigned to manage the care of high-risk patients. It was agreed that ACCs would be sent the medication review, in addition to the primary care provider (PCP), for all patients already assigned to an ACC. The ACC would then facilitate and assist the provider in implementing recommendations. For patients not assigned to an ACC, it was agreed that the pharmacist could send reviews to an ACC on a case-by-case basis, in most cases for patients with an HbAlc $\geq 8.5 \%$ and/or for patients with medication nonadherence. Providers were educated about the program through various methods, including presentations at department meetings, marketing flyers, and email reminders.

Patients lost to follow-up and patients without PCPs proved to be an obstacle to comprehensive population health management. Since the ViPRx program was designed for reviews to be completed in a previsit fashion, patients who never came to see their providers were not being reviewed. Likewise, since the program was designed in the primary care space, patients who did not have a PCP and received all care through the endocrine clinic were also not being reviewed. To address the barrier resulting from patients lost to follow-up, the pharmacy partnered with care coordination to reengage these patients. To do so, the pharmacist would complete a pvCMR for these patients and then forward the note to an ACC who would then contact the patient and encourage and assist with scheduling a follow-up visit. To address the barrier presented by patients without PCPs, the pharmacy collaborated with the endocrine clinic. It was agreed that for these patients, the ViPRx pharmacist would complete reviews before the patient's endocrine visit in the same manner as those sent to PCPs.

Results from the ViPRx program demonstrate the pharmacist's ability to achieve the desired outcomes of positively affecting clinical and cost-savings measures. In the first full year of the ViPRx program, 1 full-time pharmacist completed 871 pvCMRs, reviewed over 8,000 e-prescriptions, and made over 3,400 recommendations to improve medication use. In the second full year, 1 full-time pharmacist completed 935 pvCMRs, reviewed over 8,000 e-prescriptions, and made over 3,700 recommendations. The majority of reviews take 30-60 minutes to complete, and on average, the pharmacist makes 4 recommendations per review. The pharmacist completes up to 8 pvCMRs per day and averages 4 pvCMRs and 15 interventions per day. An additional 10-20 minutes per review is required to track acceptance of recommendations. 
Provider acceptance of recommendations steadily increased over the first 6 months of the program from 55\% to 70\%. The improved acceptance rate is indicative of improved provider acceptance of the program and increased provider confidence and trust in the pharmacist's recommendations. Recommendations most likely to be accepted included lab monitoring, medication nonadherence counseling, medication reconciliation, and increasing medication doses. A 5-month collaborative project with care management was successful in getting $70 \%$ of patients identified as lost to follow-up to reengage in care with their PCPs.

Clinical outcomes have been measured by the effect on diabetes health registry scores and medication adherence. The health registry measures were created and designed by population health IT with provider input. The measures are modeled after national standards such as the Centers for Medicare \& Medicaid Services quality measures. ${ }^{5}$ Measures for the diabetes health registry at our institution include $\mathrm{HbAlc} \leq 9 \%$, HbAlc monitoring, LDL monitoring, LDL controlled, and diabetic nephropathy screening. Screening/monitoring measures are considered "met" if they have been completed in the past year. LDL is considered "controlled" if a patient is prescribed statin therapy or has an $\mathrm{LDL}<100$.

Improvements in all diabetes registry measures have been demonstrated since the implementation of ViPRx (Figure 1). Most notable is a $6 \%$ increase in the percentage of patients with an $\mathrm{HbAlc}$ value of $9 \%$ or less and a $7 \%$ improvement in the controlled LDL measure. Monitoring parameters (nephropathy screening, HbAlc, and LDL) increased by $8 \%-12 \%$ from baseline.

To assess the effect on medication adherence, medication possession ratios (MPRs) for antidiabetic agents for patients in our population were obtained from the pharmacy benefit manager (PBM). The data revealed a 1.5\% improvement in MPR since implementation of ViPRx. Of note, within the same time frame that our custom network MPR improved by $1.5 \%$, the MPR of a similar peer group fell by $0.5 \%$. The peer group is a benchmark group defined by the PBM that includes over 100 universities and more than 700,000 patients.

Cost savings related to discontinuation of unnecessary medications and duplications of therapy has resulted in an estimated prescription medication savings of $\$ 11$ per member per month (PMPM) in the defined population. A separate cost analysis performed collaboratively by our IT, finance, and benefits departments revealed that patients in the intervention group had an overall lower PMPM trend compared with diabetes patients enrolled in either of the health system's other 2 plans, a high deductible plan and a preferred provider plan (Figure 2).

\section{FIGURE 1 Diabetes Health Registry Scores}

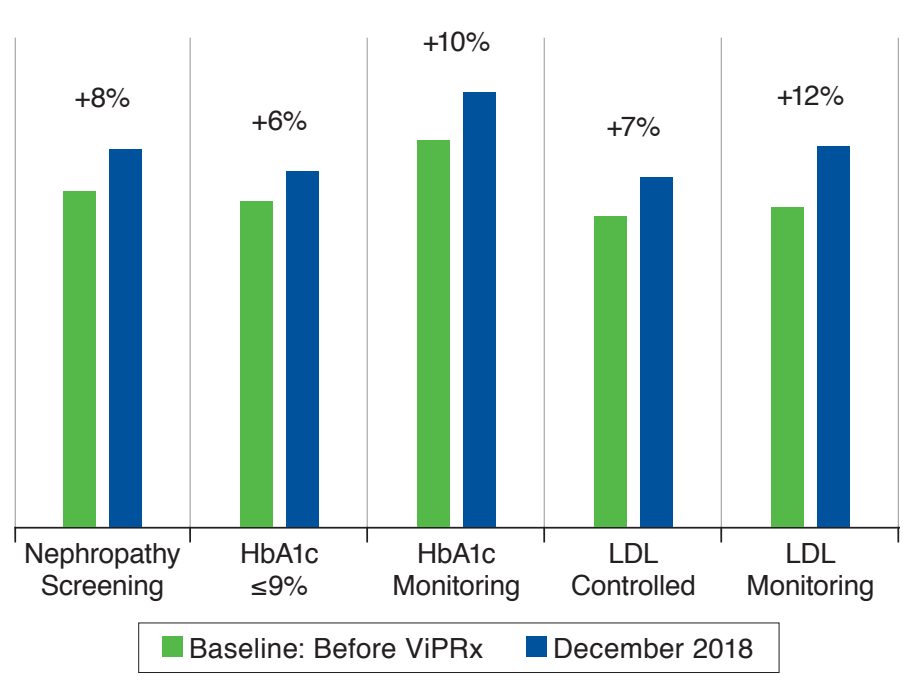

HbAlc=hemoglobin Alc; LDL=low-density lipoprotein; ViPRx=virtual pharmacy review.

\section{Implications}

The ViPRx program represents a new use of the EMR and reporting capabilities and has promoted the use of the intervention tracking capabilities within the electronic record for other pharmacy programs and initiatives. By increasingly tracking interventions electronically, the pharmacy department is able to quickly gather and present data to show the value of our interventions. Furthermore, the virtual nature of the program affords it extensive flexibility and adaptability. To that point, the health system's population health team identified a need for interventions to improve outcomes and lower costs for patients with chronic obstructive pulmonary disease (COPD). To accommodate this request, patients with custom/ narrow network insurance and a COPD diagnosis were added to the ViPRx pharmacist's patient panel. Owing to the virtual nature of the ViPRx program and its optimization of technology resources, the ViPRx program was able to add this new patient population with ease. Additional resources needed to include the COPD population were limited to updating the daily worklist to identify appointments for these patients and updating the electronic intervention tracker to include COPD. After these 2 easy updates that were complete within a few days, the ViPRx pharmacist began completing pvCMRs for COPD patients in addition to the diabetes patients.

The ViPRx program is a program that optimizes resources and relationships to position pharmacy as a key player in successful population health initiatives. The creative application of technology allows pharmacists to have a broader presence 


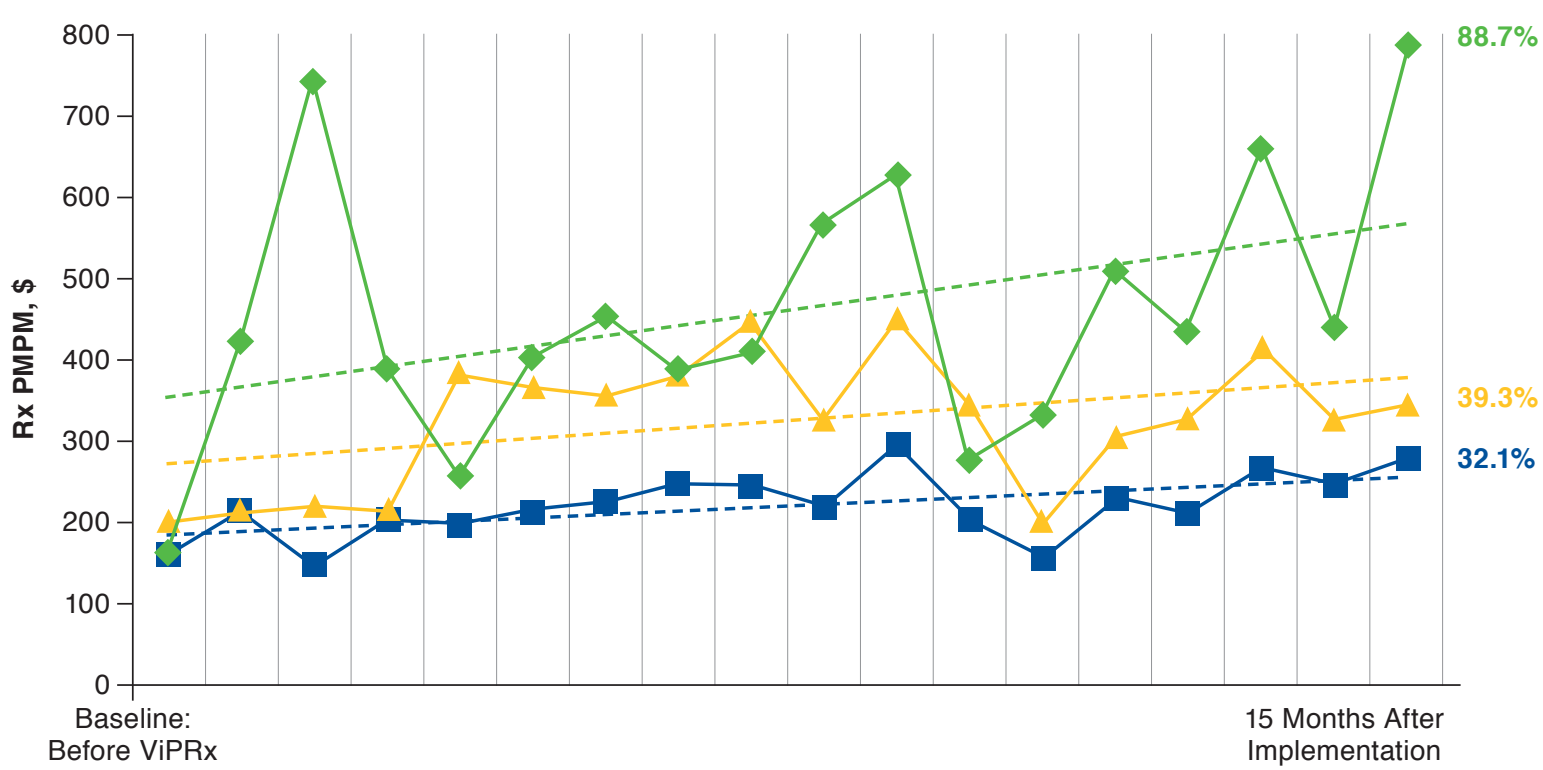

$\longrightarrow$ High-Deductible Plan $\longrightarrow$ Narrow/Custom Network Plan $\longrightarrow$ PPO Plan

PMPM = per member per month; $P P O=$ preferred provider organization; $R x=$ prescription; ViPR $=$ virtual pharmacy review.

and impact within the health system. In this case, 1 fulltime equivalent pharmacist performs pvCMRs for patients at 8 different primary care clinics simultaneously. The innovative use of technology also extends to data tracking and outcome reporting, allowing for justification of pharmacy-driven improvements in key clinical and cost-saving metrics.

The program is also original in its design and uses pharmacists as the medication experts they are but outside the realm of traditional face-to-face ambulatory care clinics or telehealth visits. By narrowing in on the pharmacist's skill as a medication expert and leveraging technology that facilitates chart review and communication to providers from any location, the program optimizes pharmacist time by allowing 1 pharmacist to cover a larger number of patients. This optimization of personnel makes the program more financially appealing and sustainable long term.

The reliance on technology allows the program the adaptability and flexibility needed to meet the needs of the health system as those needs change, as evidenced by the quick and seamless addition of COPD patients to the pharmacist's panel. The technology used also allows for transitions of care management within the program by providing the pharmacist with real-time alerts of prescribed medications at time of discharge from the hospital, emergency department, or urgent care within the same health system. Extensive collaboration with other disciplines in the health care system, including multiple IT departments, physicians, care coordinators, and finance, is another uniquely effective component of the program.

\section{Conclusions}

ViPRx is a population health management program that creatively optimizes the pharmacist's skills as a medication expert and the available technology to allow for a broader patient reach and simultaneously improved the care of patients seen across 8 primary care clinics and a specialty endocrinology clinic in the health system. The program consists of a pharmacist performing pvCMR virtually and in a timely manner, within the EMR, thus, allowing the pharmacist to affect patient care in collaboration with the patient's established PCP or endocrinologist.

The effect of the ViPRx program is best described as "high volume" and "high impact." One pharmacist is able to complete more reviews and make more interventions because of the virtual nature of the reviews without compromising outcomes. Implementation of the ViPRx program has resulted in improvements in clinical and financial measures. ViPRx is an innovatively designed program that advances pharmacy practice by demonstrating a significant benefit of incorporating a pharmacist into population health initiatives within the health system. 


\section{Authors}

BUSHRA MURAYWID, PharmD, BCPS; LAURA E. BUTKIEVICH, PharmD, MHA, BCPS; and BRAD MYERS, PharmD, MBA, BCPS, University of Missouri Health Care, Columbia.

AUTHOR CORRESPONDENCE: Bushra Muraywid, PharmD, BCPS, Clinical Pharmacy Specialist, Ambulatory Care/Population Health, University of Missouri Health Care, One Hospital Dr., DC060.00, Columbia, MO 65212. Tel.: 573.882.5025;

E-mail: muraywidb@health.missouri.edu.

\section{DISCLOSURES}

No outside funding supported this work. The authors have no conflicts of interest to disclose.

This work was presented at the 2017 Vizient Connections Summit; April 6, 2017; Las Vegas, NV, and the 2018 Cerner Health Conference; October 10, 2018; Kansas City, MO.

\section{ACKNOWLEDGMENTS}

The authors thank Ryan Roark, Data Architect, at Cerner Corporation (Tiger Institute), for assisting with data reporting.

\section{REFERENCES}

1. Kindig D, Stoddart G. What is population health? Am J Public Health. 2003;93(3):380-83.

2. Chisholm-Burns MA, Kim Lee J, Spivey CA, et al. US pharmacists' effect as team members on patient care: systematic review and meta-analyses. Med Care. 2010;48(10):923-33.

3. Brummel A, Lustig A, Westrich K, et al. Best practices: improving patient outcomes and costs in an ACO through comprehensive medication therapy management. J Manag Care Spec Pharm. 2014;20(12):1152-58. Available at: https://www.jmcp.org/doi/abs/10.18553/jmcp.2014.20.12.1152.

4. Cutler RL, Fernandez-Llimos F, Frommer M, et al. Economic impact of medication non-adherence by disease groups: a systematic review. BMJ Open. 2018;8(1):e016982. Available at: https://bmjopen.bmj.com/content/ bmjopen/8/1/e016982.full.pdf. Accessed December 12, 2019.

5. eCQI Resource Center, Office of the National Coordinator for Health Information Technology. Electronic clinical quality measures for eligible professionals and eligible clinicians: 2019 reporting [table]. Updated November 2018. Available at: https://ecqi.healthit.gov/system/files/EP_EC_ MeasuresTable_2018-05_v3.pdf. Accessed December 12, 2019. 